\section{Panorama de la dependencia en Chile: avances y desafíos}

\author{
PABLO VILLALOBOS DINTRANS ${ }^{1, a}$
}

\section{Dependency in Chile. Advances and challenges}

Chile is facing a process of rapid aging which poses several challenges. Among these challenges is the increase of dependency in the population. Despite its relevance, the topic has not been prioritized in the agenda. One explanation for this low-priority is the lack of information on the concept of dependency. This article shows that, in fact, no consensus exists in Chile on how to define and measure dependency. Additionally, it provides an updated estimation of dependency in the country, aiming to foster further debate on the topic.

(Rev Med Chile 2019; 147: 83-90)

Key words: Disabled Persons; Dependency (Psychology); Long-Term Care.

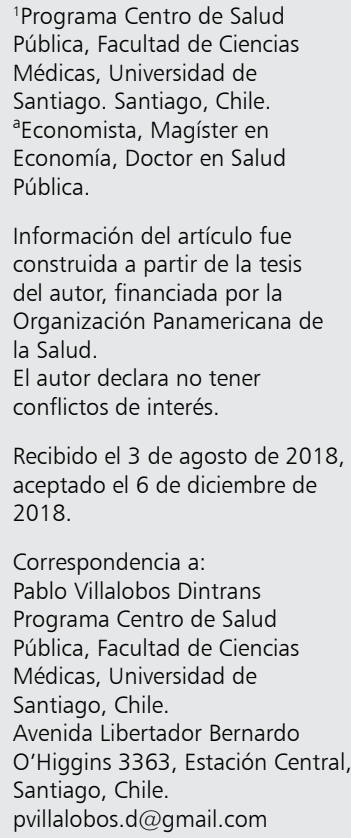

Información del artículo fue construida a partir de la tesis del autor, financiada por la Organización Panamericana de la Salud.

El autor declara no tener conflictos de interés.

Recibido el 3 de agosto de 2018, aceptado el 6 de diciembre de 2018.

Correspondencia a:

Pablo Villalobos Dintrans Programa Centro de Salud Pública, Facultad de Ciencias Médicas, Universidad de

Santiago, Chile.

Avenida Libertador Bernardo

O’Higgins 3363, Estación Central,

Santiago, Chile.

pvillalobos.d@gmail.com

C hile está en un proceso de rápido envejecimiento que presenta diversos desafíos. A pesar de la urgencia por responder a estos cambios, estudios recientes señalan que el país no está preparado para afrontar esta nueva etapa ${ }^{1,2}$. Un tema pendiente es el de los cuidados de largo plazo, concepto que se encuentra estrechamente ligado al incremento del número de personas con dependencia en el país.

Una de las principales barreras para avanzar en el tema de la dependencia y los cuidados es la falta de definiciones básicas y estadísticas. En general, los cuidados de largo plazo han sido considerados como una problemática privada, que ocurre al interior de los hogares y que permanece invisible a ojos de la sociedad; este enfoque ha impedido la generación de información al respecto, dificultando el diseño de políticas públicas para abordarlo³. De manera similar, el modelo de ventanas de oportunidad de Kingdon ${ }^{4}$ plantea que para que un tema logre entrar en la agenda requiere ser identificado como un problema; la falta de información sobre la dependencia hacen que, a pesar de su importancia, el tema no se priorice en el país.

Existen múltiple definiciones de dependencia. Por ejemplo, para Chile, se podrían considerar las provenientes de la legislación chilena ${ }^{5}$, el Estudio Nacional de la Dependencia en Adultos Mayores realizado por el Servicio Nacional del Adulto Mayor (SENAMA) ${ }^{6}$ y el reporte de la Organización Mundial de la Salud sobre envejecimiento y salud ${ }^{7}$. Es importante notar que a pesar de sus diferencias, las definiciones identifican como crucial la existencia de limitaciones para realizar ciertas actividades y la necesidad explícita de ayuda de terceros. Sin embargo, la operacionalización de estas definiciones teóricas representa un reto, tanto por la falta de una definición de consenso como por la multiplicidad de instrumentos para medir dependencia.

El objetivo de este artículo es presentar un panorama de la dependencia en Chile, incluyendo el camino recorrido hasta la fecha y el estado actual de la dependencia en el país, con el fin de estructurar y fomentar la discusión en el tema.

\section{Materiales y Métodos}

El análisis se divide en dos partes. En una primera parte, se realiza una búsqueda de artículos que han intentado medir la dependencia en el 
país, a fin de mostrar la evolución del concepto y las estimaciones realizadas en los últimos años. En segundo lugar, se realiza un análisis de la dependencia en Chile hoy, usando la Encuesta de Caracterización Socioeconómica Nacional (CASEN) del año 2015. El análisis busca mostrar el camino recorrido en el país para responder la pregunta: ¿cuántos dependientes hay en Chile?

Con el fin de mostrar las dificultades en la medición del concepto de dependencia, se realizó una búsqueda de estudios que han intentado cuantificar el concepto en los últimos años. El listado de estudios se generó a partir de una búsqueda en línea (Pubmed, Google Scholar y Google), usando "dependencia Chile" y "medición dependencia Chile" como palabras claves. La búsqueda incluyó documentos en español publicados entre los años 2000 y 2017. Adicionalmente, se usó la metodología de snowball sampling -buscando referencias dentro de los documentos ya encontrados-para incluir investigaciones que pudieran cumplir con los criterios de inclusión. Finalmente, la búsqueda se complementó con un proceso de consulta a expertos para identificar estudios en la literatura gris. Como criterio de inclusión, se utilizó la población estudiada, considerando solo estudios que usan encuestas con representatividad nacional, encuestas realizadas en el Gran Santiago y encuestas en población adulta, excluyendo estudios en poblaciones más acotadas. El listado de los estudios se presenta en el Anexo 1.

El análisis estadístico se basó en la última encuesta CASEN, del año 2015. El uso de esta base permite obtener resultados con representatividad nacional y replicar el análisis a futuro. La población con dependencia fue identificada a partir de las definiciones establecidas por SENAMA ${ }^{6}$.

\section{Resultados}

\section{Estudios de dependencia en Chile}

Una primera observación a partir del listado de estudios del Anexo 1 es su alta heterogeneidad. Los estudios usan distintas fuentes de información, se enfocan en distintas poblaciones y utilizan distintas definiciones e instrumentos para medir la dependencia. Todo lo anterior dificulta la comparabilidad de los resultados y pone de manifiesto la falta de consenso respecto del significado de la dependencia y su medición.
$\mathrm{Al}$ analizar el listado de manera cronológica, se observa que los estudios de principio de la década 2000-2010 se enfocaban en temas de envejecimiento y discapacidad, conceptos relacionados, pero no sinónimos de dependencia. El desafío de la dependencia es identificar algún grado de pérdida de funcionalidad en los individuos, entendida como una interacción entre el individuo, su condición y su entorno. Muchos estudios han intentado abordar este problema usando distintas definiciones de dependencia, que van desde la existencia de un cuidador, a la aplicación de pruebas para medir funcionalidad, principalmente basados en los conceptos de actividades básicas de la vida diaria (ABVD) y actividades instrumentales de la vida diaria (AIVD).

Así, por ejemplo, las encuestas nacionales de discapacidad ${ }^{8,9}$ entregan estimaciones de la discapacidad en el país, que pueden ser consideradas como una cota superior para la estimación de la dependencia. De acuerdo a estos datos, el porcentaje de discapacitados estaría entre $13 \%$ y $17 \%$ de la población, porcentaje que se incrementa con la edad, siendo cercano a $40 \%$ en adultos mayores (AM). En línea con un mayor entendimiento e importancia del concepto de dependencia, la encuesta más reciente ${ }^{9}$ incluye estimaciones de dependencia que muestran que solo un subconjunto de los discapacitados $(40,4 \%)$ presentan algún grado de dependencia, representando cerca de $8 \%$ de la población total del país.

La Encuesta Nacional de Salud ${ }^{10,11}$, usando un set de definiciones e instrumentos distintos, encuentra que el porcentaje de personas con discapacidad sería cercano a $7 \%{ }^{11}$; también se indica el porcentaje de personas mayores de 60 años con deterioro cognitivo se situaría entre $4 \%$ y $10 \%$ de la población, similar a lo reportado en el estudio SABE realizado en Santiago ${ }^{12}$.

Otro grupo de estudios intentan avanzar en la medición de dependencia usando como métrica el porcentaje de la población que reporta dificultades para realizar ABVD y AIVD ${ }^{12-14}$. Si bien estos porcentajes varían dependiendo del tipo de actividad evaluada, los estudios encuentran que el porcentaje de personas que reportan tener dificultad para realizar algunas actividades crece con la edad y también es mayor entre personas con menor nivel de ingresos, enfatizando la interacción entre la condición de salud de los individuos y su entorno.

Finalmente, una serie de estudios más recientes 
intentan medir dependencia directamente ${ }^{6,15-17}$. En esta línea, el Estudio Nacional de la Dependencia en Adultos Mayores ${ }^{6}$ marca un punto de inflexión respecto de la definición y medición de la dependencia en el país. El estudio elabora un instrumento específico para medir la dependencia en AM en Chile. Los resultados muestran que $24 \%$ de la población mayor de 60 años presenta algún grado de dependencia, con más de $50 \%$ de los dependientes clasificados como severos. Similares resultados se obtienen de otros estudios usando diversas fuentes, en donde también se constata que tanto la prevalencia como la severidad de la dependencia aumentan con la edad ${ }^{15-17}$. Por último, la encuesta CASEN ha incorporado preguntas que permiten caracterizar la dependencia basada en ABVD y AIVD. A diferencia del resto de los estudios, la encuesta no se restringe a los AM, lo que permite extender el análisis a la población general. A pesar de esto, los documentos publicados por el Ministerio de Desarrollo Social (MIDESO) limitan el análisis a la población adulta mayor ${ }^{18,19}$. Los resultados se asemejan a los encontrados en otros estudios, donde el porcentaje de población adulta mayor con dependencia fluctúa en torno a $20 \%$. A pesar que el análisis de ambas encuestas (CASEN 2013 y 2015) se basan en la metodología propuesta por SENAMA ${ }^{6}$, sus resultados no son comparables $^{19}$.

Finalmente, datos de encuestas recientes muestran que el porcentaje de cuidadores en la población se situaría entre 5 y el $10 \%{ }^{20}$, mientras que las estimaciones realizadas por Ministerio de Desarrollo Social, en el contexto del Sistema Nacional de Cuidados (SNAC), muestran que el porcentaje de dependientes en la población estaría en torno a $2 \%{ }^{21}$.

El ejercicio de analizar la evolución histórica de los esfuerzos por medir la dependencia en Chile es útil en varios aspectos. Primero, para ejemplificar las dificultades prácticas de llevar a cabo esta medición; segundo, para entender el cambio de énfasis producido a lo largo del tiempo, desde una mirada acotada al envejecimiento y la discapacidad, hacia el surgimiento del concepto de dependencia; tercero, para evidenciar que, a pesar de una aparente convergencia de los estudios respecto del uso del concepto de dependencia, todavía existe una falta de consenso respecto de cómo medirlo. Lo anterior es relevante, ya que, como muestra el Anexo 1, a pesar de la gran cantidad de informa- ción disponible, aún no es posible tener una cifra respecto de la magnitud del problema en el país y los datos de los distintos estudios no son comparables, dificultando el análisis de la evolución del tema a lo largo del tiempo.

\section{Análisis de la dependencia usando base de datos CASEN 2015}

La encuesta CASEN se realiza periódicamente desde el año 1990 con el fin de conocer la situación socioeconómica de los hogares del país y evaluar el impacto de la política social. A partir del año 2006, su cuestionario incluye preguntas que permiten identificar discapacidades y ha incorporando en sus últimas versiones preguntas que permiten identificar dependencia ${ }^{22}$. La evolución de la encuesta CASEN refleja el camino seguido por varias instituciones en tratar de entender, definir y medir el concepto de dependencia (Tabla 1).

En su última versión, la encuesta usa las definiciones establecidas por SENAMA ${ }^{6}$, mostrando la intención de medir dependencia directamente. Los datos presentados provienen de la versión 2015 de la encuesta.

La Figura 1 muestra la prevalencia de la dependencia en distintos grupos de edad. En primer lugar, se observa que la prevalencia (porcentaje de dependientes en cada grupo de edad) aumenta con la edad, superando el $10 \%$ de la población a partir de los 70 años.

La Tabla 2 muestra que, usando la definición de dependencia propuesta por SENAMA y MI$\mathrm{DESO}^{6,19}$, el número de dependientes en el país alcanzaría a 625.484 personas, aproximadamente $4,5 \%$ de la población mayor de 15 años. De estos, $63,40 \%$ son mujeres y $36,60 \%$ son hombres.

Respecto de la prevalencia por sexo, los datos muestran que no solo el total de dependientes son mayoritariamente mujeres, sino, además, que la prevalencia de la dependencia es mayor en este grupo: mientras 3,53\% del total de hombres mayores de 15 años en el país es clasificado como dependiente funcional, esta cifra es 5,29\% para las mujeres.

La Figura 1 muestra que la dependencia es particularmente prevalente en AM, lo que ha justificado la priorización de este grupo en estudios y políticas. Sin embargo, es importante destacar que, si bien la dependencia es más prevalente en AM, buena parte de los dependientes son menores de 65 años: si bien la prevalencia es baja en este grupo 


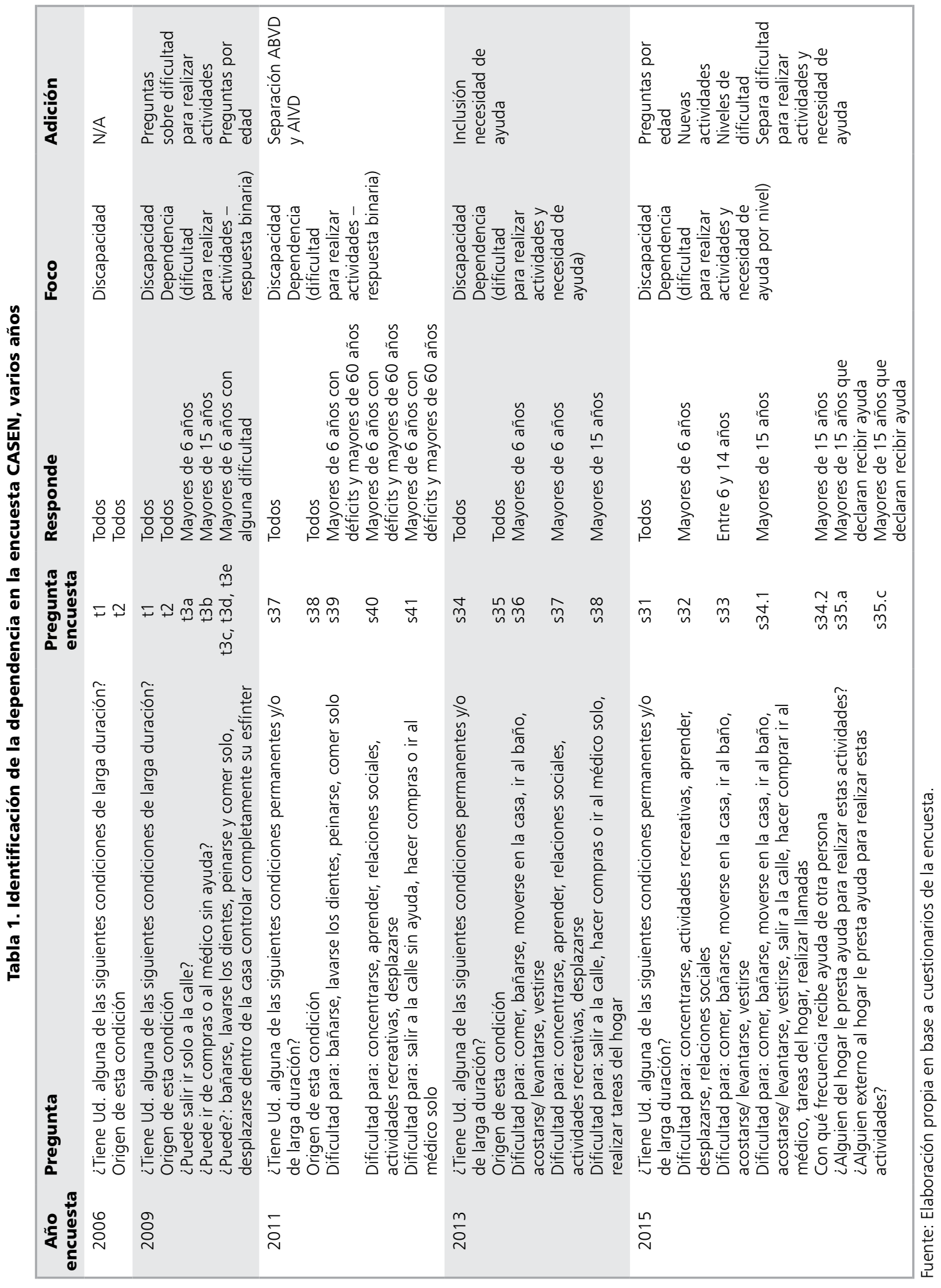




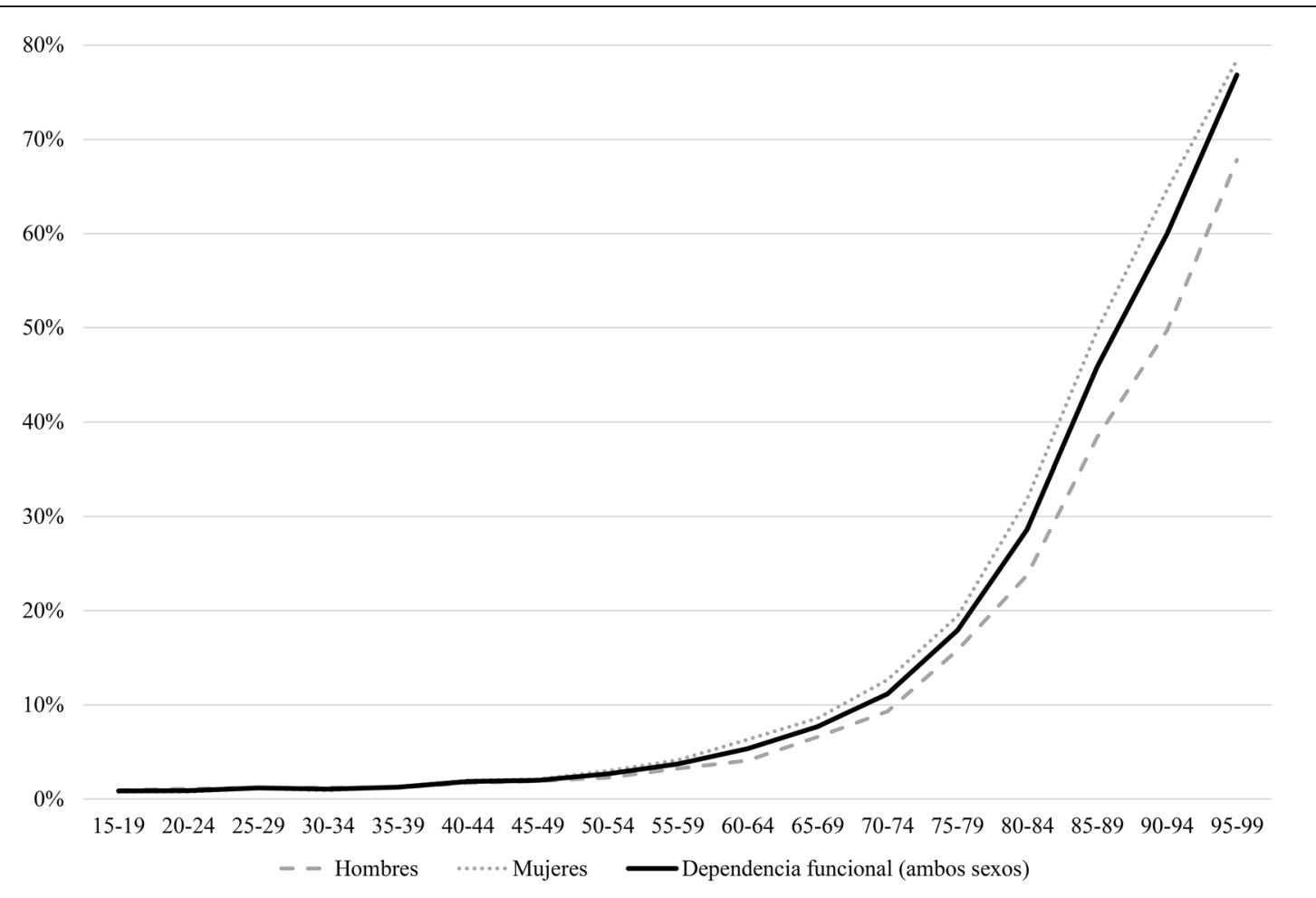

Figura 1. Prevalencia de la dependencia en mayores de 15 años por grupos de edad, Chile (2015). Nota: Elaboración propia en base a CASEN 2015.

Tabla 2. Estadísticas de dependencia en Chile, 2015

\begin{tabular}{|c|c|c|c|c|}
\hline $\begin{array}{l}\text { Número de } \\
\text { dependientes }\end{array}$ & $\begin{array}{l}\text { Prevalencia en la } \\
\text { población totala }\end{array}$ & $\begin{array}{l}\text { Prevalencia en } \\
\text { mayores de } \\
15 \text { años }^{\mathbf{b}}\end{array}$ & $\begin{array}{l}\text { Prevalencia en } \\
\text { mayores de } \\
65 \text { años }\end{array}$ & $\begin{array}{c}\text { Porcentaje de } \\
\text { dependientes } \\
\text { mayores de } 65 \text { años }\end{array}$ \\
\hline 625.484 & 3,56 & 4,47 & 18,00 & 63,83 \\
\hline
\end{tabular}

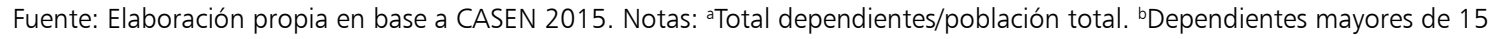
años/población mayor de 15 años. 'Dependientes mayores de 65 años/población mayor de 65 años. 'Dependientes mayores de 65 años/total dependientes.

etario, estos representan casi $90 \%$ del total de la población. Lo anterior implica que una política enfocada exclusivamente en AM, excluye a casi $40 \%$ de los dependientes del país.

En cuanto al nivel de dependencia, la encuesta permite clasificar a los dependientes en leves, moderados y severos, usando la metodología de SENAMA y MIDESO ${ }^{6,18,19}$. Como muestra la Figura 2, la distribución por nivel de dependencia es relativamente equitativa (en torno a $30 \%$ ) para todos los grupos de edad hasta los 80 años: consi- derando el total de dependientes, $33 \%$ presentan dependencia leve, $43 \%$ moderada y $24 \%$ severa. Sin embargo, se observa que la dependencia severa es mucho más prevalente en edades tempranas (entre 15 y 25 años) y a partir de los 80 años, edad en la que el porcentaje de dependientes leves se reduce de manera importante.

En resumen, la encuesta CASEN muestra que la población (mayor de 15 años) que tiene algún grado de dependencia en el país se sitúa en torno a las 625 mil personas, mayoritariamente concentra- 


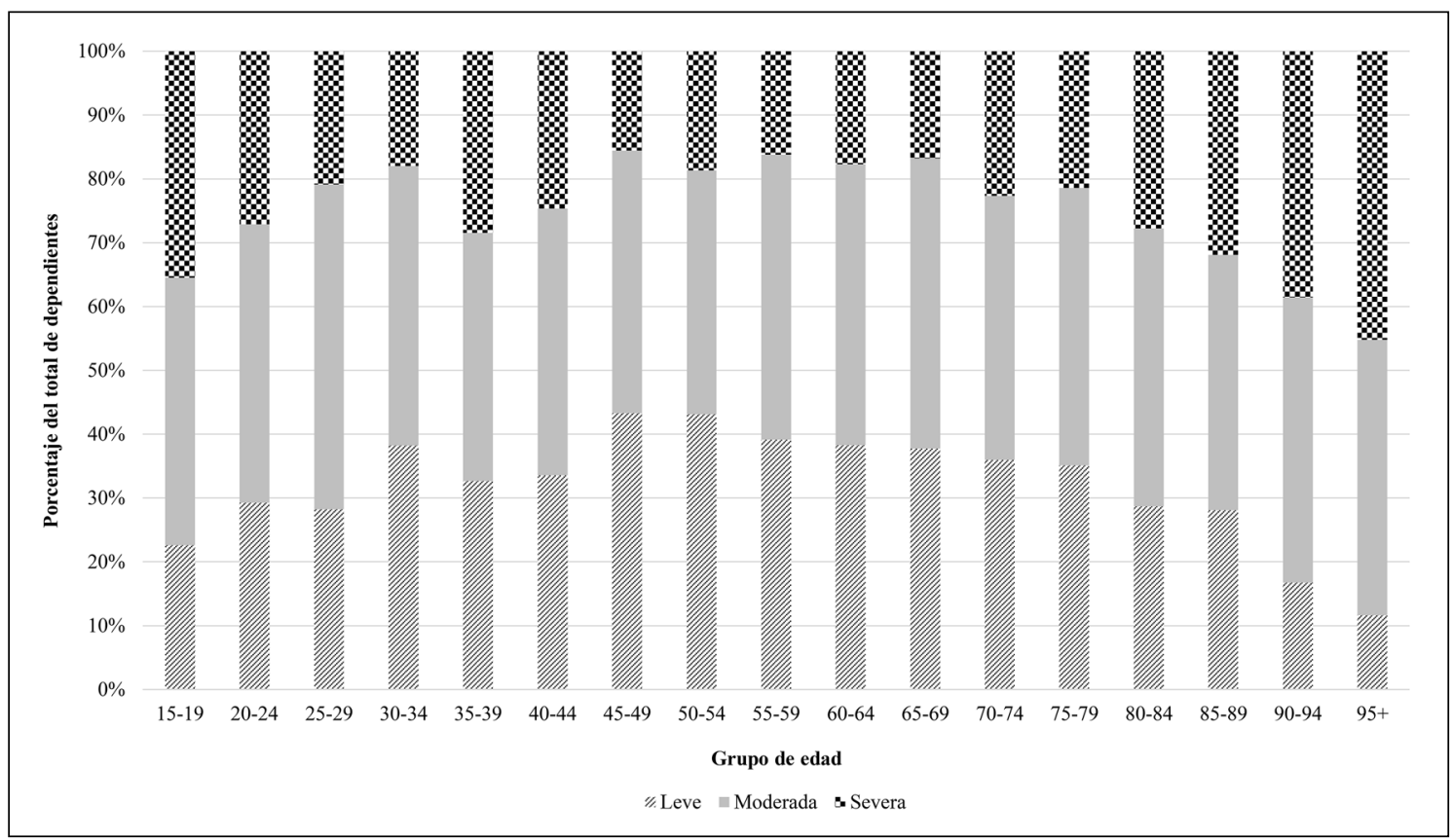

Figura 2. Dependencia por nivel y grupo etario, Chile (2015). Nota: Elaboración propia en base a CASEN 2015.

da en AM. Los datos muestran que la prevalencia de la dependencia aumenta progresivamente con la edad, incrementándose abruptamente a partir de los 70 años. A partir de esta edad, también aumenta su nivel de severidad.

\section{Discusión}

El análisis presentado muestra cómo el país ha ido avanzando en relevar el tema de la dependencia, generando información, primero relacionada con discapacidad y luego con funcionalidad, en distintas encuestas y estudios. También se observa el camino recorrido en cuanto a tratar de conceptualizar y medir un término sobre el que no existe un consenso claro ni un instrumento único de medición. En este sentido, la experiencia internacional muestra la necesidad de avanzar en una definición única para la dependencia, así como de una metodología específica para medirla ${ }^{23-29}$.

Los estudios para Chile coinciden en que la prevalencia de la dependencia aumenta con la edad, especialmente en AM. Finalmente, es necesario destacar que si bien la dependencia se concentra en los AM, está lejos de estar restringida a este grupo de edad: aproximadamente $40 \%$ de los dependientes tienen menos de 65 años, por lo que si bien la focalización en este grupo tiene sentido, se requiere pensar en una manera de incorporar dependientes de todas las edades en las políticas públicas.

Los resultados a partir de la encuesta CASEN permiten estimar los dependientes en el país, pero deben ser interpretados con cuidado. En primer lugar, es necesario considerar que la encuesta usa la metodología propuestas por SENAMA ${ }^{6}$, pero excluye la identificación de demencia, lo que implica una subestimación de los dependientes. Ignorar las enfermedades mentales, particularmente demencia, significa desconocer parte importante del problema, especialmente considerando que la demencia ha sido identificada como la principal causa de dependencia en AM en el mundo y un importante contribuyente a la pérdida de funcionalidad en Chile $^{30}$. Por otra parte, los datos de la encuesta son autorreportados, lo que genera una segunda fuente de potencial error en la medición.

El análisis da cuenta de una importante brecha respecto de la medición de la dependencia en Chile, ya que no existe una definición e instrumento únicos, actualmente no es posible contar con una 
estimación precisa del problema. Sin embargo, el artículo también enfatiza los progresos en la materia durante los últimos años. En esta línea, destaca la necesidad de avanzar hacia el establecimiento de un instrumento que permita medir la dependencia en toda la población, continuando el hito marcado por el Primer Estudio Nacional de la Dependencia $^{6}$. La incorporación de esta metodología en la encuesta CASEN representa un importante logro en este sentido.

En esta línea, es necesario trabajar en la oficialización, a nivel nacional, de una medición de dependencia que permita identificar esta población y que pueda servir como instrumento para selección y clasificación de beneficiarios en un futuro sistema de cuidados de largo plazo.

\section{Referencias}

1. Thumala D, Kennedy BK, Calvo E, González-Billault C, Zitko P, Lillo P, et al. Aging and health policies in Chile: new agendas for research. Health Systems \& Reform 2017; 3 (4): 253-60.

2. Villalobos Dintrans P. Envejecimiento y cuidados a largo plazo en Chile: desafíos en el contexto de la OCDE. Rev Panam Salud Publica 2017; 41: e86.

3. Scheil-Adlung X. Long-term care protection form older persons: A review of coverage deficits in 46 countries. ESS Working Paper 50, International Labour Office; 2015.

4. Kingdon JW. Agendas, Alternatives, and Public Policies, 2nd Edition. Boston: Pearson; 1995.

5. Biblioteca del Congreso Nacional. Ley 20.422 Establece normas sobre igualdad de oportunidades e inclusión social de personas con discapacidad. Santiago: Biblioteca de Congreso Nacional; 2018. Disponible en: https:// www.leychile.cl/Navegar?idLey=20422. (Acceso: 4 de julio de 2018).

6. Servicio Nacional del Adulto Mayor. Estudio Nacional de la Dependencia en la Personas Mayores. Santiago: SENAMA; 2010. Disponible en: http://www.senama. $\mathrm{cl} /$ filesapp/Estudio\%20Nacional\%20de\%20Dependencia\%20en\%20las\%20Personas\%20Mayores.pdf. (Acceso: 4 de julio de 2018).

7. World Health Organization. World Report on Ageing and Health. Geneva: World Health Organization; 2015.

8. Fondo Nacional de la Discapacidad. Primer Estudio Nacional de la Discapacidad en Chile. Santiago: FONADIS; 2014. Disponible en http://www.senadis.gob.cl/ pag/136/1196/resultados_endisc_i. (Acceso: 4 de julio de 2018).
9. Ministerio de Desarrollo Social. Segundo Estudio Nacional de la Discapacidad en Chile. Santiago: MIDESO; 2015. Disponible en http://www.senadis.gob. cl/pag/355/1197/ii_estudio_nacional_de_discapacidad (Acceso: 4 de julio de 2018).

10. Ministerio de Salud. Encuesta Nacional de Salud ENS Chile 2003. Santiago: MINSAL; 2003. Disponible en http://www.medicinadefamiliares.cl/Protocolos/encnacsalres.pdf (Acceso: 3 de julio de 2018).

11. Ministerio de Salud. Encuesta Nacional de Salud ENS Chile 2009-2010. Santiago: MINSAL; 2010. Disponible en http://web.minsal.cl/portal/url/item/bcb03d7bc28b64dfe040010165012d23.pdf (Acceso: 4 de julio de 2018).

12. Organización Panamericana de la Salud. Salud, bienestar y envejecimiento en Santiago, Chile. SABE 2000. Santiago: OPS; 2005. Disponible en http://iris.paho.org/ xmlui/bitstream/handle/123456789/741/9275316090. pdf? sequence $=1$ (Acceso: 4 de julio de 2018).

13. Ministerio de Salud. II Encuesta de Calidad de Vida y Salud. Santiago: MINSAL; 2006. Disponible en http:// www.crececontigo.gob.cl/wp-content/uploads/2015/11/ ENCAVI-2006.pdf (Acceso: 3 de julio de 2018).

14. Ministerio de Desarrollo Social. Casen 2013 - Inclusión social de personas con dificultades y/o condiciones permanentes y de larga duración. Santiago: MIDESO; 2015. Disponible en http://observatorio.ministeriodesarrollosocial.gob.cl/documentos/Casen2013_inclusion_social. pdf (Acceso: 3 de julio de 2018).

15. Olivares-Tirado P, Ibáñez C, España M, Miranda V, Gillmore M. Dependencia en adultos mayores en Chile. Documento de Trabajo Superintendencia de Salud; 2008. Disponible en http://www.supersalud.gob.cl/documentacion/666/articles-4471_recurso_1.pdf (Acceso: 3 de julio de 2018).

16. Cid C, Marín PP, Starck N, Marcó C, Clavero C, López D, Romero A. Informe Final Análisis Prospectivo de un Seguro Obligatorio de Dependencia para Adultos Mayores. Santiago: Subsecretaría de Previsión Social; 2014.

17. Ministerio de Salud. Programa Nacional de Salud de las personas adultas mayores. Santiago: MINSAL; 2014. Disponible en http://web.minsal.cl/sites/default/files/ files/Borrador\%20documento\%20Programa $\% 20$ Nacional\%20de\%20Personas\%20Adultas\%20Mayores-\%20 04-03_14.pdf (Acceso: 3 de julio de 2018).

18. España M. Panorama CASEN: Dependencia funcional en las personas mayores. Santiago: MIDESO; 2016. Disponible en http://observatorio.ministeriodesarrollosocial.gob.cl/documentos/Panorama_Casen_Dependencia_funcional_personas_mayores_corr.pdf (Acceso: 5 de julio de 2018).

19. Ministerio de Desarrollo Social. CASEN 2015 - Adultos 
Mayores: Síntesis de Resultados. Santiago: MIDESO; 2017. Disponible en http://observatorio.ministeriodesarrollosocial.gob.cl/casen-multidimensional/casen/ docs/CASEN_2015_Resultados_adultos_mayores.pdf (Acceso: 4 de julio de 2018).

20. Ministerio de Salud. Manual de Uso de Base de Datos "Tercera Encuesta de Calidad de Vida y Salud ENCAVI 2015-2016". Santiago: Ministerio de Salud; 2017.

21. Ministerio de Desarrollo Social. Minuta Estimación de Población Potencial del Subsistema Nacional de Apoyos y Cuidados. Ministerio de Desarrollo Social; 2017.

22. Ministerio de Desarrollo Social. Encuesta CASEN. Cuestionarios. Santiago: MIDESO; 2018. Disponible en: http://observatorio.ministeriodesarrollosocial.gob. cl/casen/casen_cuestionario.php. Acceso 5 julio 2018.

23. Arntz M, Sacchetto R, Spermann A, Steffes S, Widmaier $\mathrm{S}$. The German social long-term care insurance-structure and reform options. IZA Discussion Paper 2625; 2007.

24. Federal Ministry of Health. Report by the Advisory Board to Review the Definition of the Need for LongTerm Care. Bonn: Federal Ministry of Health; 20009. Disponible en https://www.bundesgesundheitsministerium.de/fileadmin/Dateien/3_Downloads/E/Englische_Dateien/Report_by_the_advisory_board_to_review_the_definition_of_the_need_for_long-term_care. pdf (Acceso: 4 de julio de 2018).
25. Federal Ministry of Health. Long-term care. Bonn: Federal Ministry of Health; 2014. Disponible en http://www. bundesgesundheitsministerium.de/en/long-term-care. html (Acceso: 4 de julio de 2018).

26. Kroneman M, Boerma W, van den Berg M, Groenewegen P, de Jong J, van Ginneken E. Netherlands: Health System Review. Health Systems in Transition 2016; 18 (2): 1-240.

27. MPHWS. Healthcare in the Netherlands. Ministry of Public Health, Welfare and Sports; 2016.

28. NHIS. National Health Insurance System of Korea: Universal Health Coverage for the World. Seoul: National Health Insurance System, Republic of Korea; 2014.

29. NHIS. Long-Term Care Insurance: Introduction. Seoul: National Health Insurance System; 2017. Disponible en https://www.nhis.or.kr/static/html/wbd/g/a/wbdga0501. html (Acceso: 6 de julio de 2018).

30. Fuentes $\mathrm{P}$, Albala C. An update on aging and dementia in Chile. Dement Neuropsychol 2014; 8 (4): 317-22.

31. World Health Organization. ICF International Classification of Functioning, Disability and Health. Geneva: World Health Organization; 2001.

32. Instituto Nacional de Estadísticas. Estadísticas Demográficas y Vitales. Santiago: INE; 2018. Disponible en: http://www.ine.cl/estadisticas/demograficas-y-vitales (Acceso: 4 de julio de 2018). 